\title{
Relación Entre Los Niveles Motivacionales Y La Autoeficacia En Estudiantes De Instrumentos De Cuerda Frotada De Nivel Superior
}

\author{
Francisco Javier Zarza Alzugaray, Dr. \\ Universidad Complutense de Madrid, Spain
}

Doi: 10.19044/esj.2018.v14n8p85 URL:http://dx.doi.org/10.19044/esj.2018.v14n8p85

\begin{abstract}
Motivation is one of the most important aspects in the development of a career of learning, even more so if one fits in a musical studies in which motivational levels must be sustained for many years. In this sense, the study of the motivation of musical achievement from the motivational theory of Weiner (2000) and its possible relation with the general self-efficacy described by Bandura (1986) is key to understanding both the formative process of the students and the possible ones pedagogical improvements to include in the Spanish educational system. Significant relationships have been found between the different motivational factors and self-efficacy. In the same way, the motivational factors related to the specific areas present a good explanatory capacity of self-perceived self-efficacy by the students. It is necessary to expand to other psychological constructs for a better understanding of the motivational event in higher musical studies.
\end{abstract}

Keywords: Music education, musical achievement motivation, self-efficacy, higher music studies

\section{Resumen}

La motivación resulta uno de los aspectos más importantes en el desarrollo de una carrera de aprendizaje, más aún si cabe en una como los estudios musicales en la que los niveles motivacionales deben estar sostenidos durante largos años. En este sentido el estudio de la motivación de logro musical a partir de la teoría motivacional de Weiner (2000) y su posible relación con la autoeficacia general descrita por Bandura (1986) resulta clave para entender tanto el proceso formativo de los estudiantes como las posibles mejoras pedagógicas a incluir en el sistema educativo español. Se han encontrado relaciones significativas entre los distintos factores motivacionales y la autoeficacia. Del mismo modo, los factores motivacionales relacionados con las áreas específicas presentan una buena capacidad explicativa de la 
autoeficacia autopercibida por los estudiantes. Se hace necesaria la ampliación a otros constructos psicológicos para una mejor comprensión del hecho motivacional en los estudios musicales superiores.

Palabras-clave: Educación musical, motivación de logro musical, autoeficacia, estudios superiores de música

\section{Introducción}

La motivación de logro está considerada como un prerrequisito para el éxito, no sólo el académico, sino también en disciplinas deportivas y situaciones relacionadas con el trabajo. En el ámbito académico, el interés en la motivación está en parte inspirado por la idea de que la motivación de los estudiantes, conformada por la sensación de competencia y creencias de valor; puede ser más maleable que la propia habilidad cognitiva, así como resultar una guía potencial para el sistema educativo a la hora de incrementar el aprendizaje y los logros de los estudiantes (Spinath, Spinath, Harlaar, y Plomin, 2006).

Existen investigaciones que diferencian, dentro de la motivación, hasta seis variables diferentes (perspectivas futuras, necesidad de logro, metas de aprendizaje, expectativa-valor, autoeficacia y autodeterminación) como moduladoras de la motivación en términos generales, como predictoras de, en última instancia, unos resultados o logros académicos. Se considera que las perspectivas futuras, la autoeficacia, la motivación ante el logro, y la expectativa-valor son unos potentes predictores del resultado académico (Elias, Mustafa, Roslan y Noah, 2011).

Aunque el logro académico es importante, en los últimos años se ha ido incrementando el conocimiento y el interés empírico en diferentes constructos psico-educacionales que pueden ser considerados como fundamentales en el ámbito educativo (Green, Liem, Martin, Colmar, Marsh y McInerney, 2012). Éstos incluyen la motivación (Pintrich, 2003), el autoconcepto (Marsh, 2007) o el compromiso con la tarea (Skinner, Kindermann, Connell y Wellborn, 2009).

La expectativa de consecución de metas, variable indispensable en la motivación de logro, se ha relacionado con variables atribucionales como la capacidad, la facilidad o dificultad de la tarea y la mejor o peor planificación del esfuerzo, la competencia y la información externa proveniente de otros (Atkinson, 1964; Heider, 1958; Manassero y Vázquez, 1998).

La teoría de la autoeficacia de Bandura (1986) y la teoría atribucional de Weiner $(1976,2000)$ han contribuido de manera notable a la comprensión y explicación de las creencias de los estudiantes sobre los logros que consiguen y sin embargo han sido raramente estudiados conjuntamente como 
modo de explicación de los posibles logros de los estudiantes (Hsieh y Schallert, 2008).

Por todo lo anterior, entendemos que variables como la motivación de logro y la autoeficacia estén relacionadas de forma directa, puesto que las concepciones que se posean de la autoeficacia específica para la consecución de una determinada meta, influirán de forma notable en la motivación exhibida hacia el logro de la tarea en cuestión. Además, el estilo atribucional en el caso de la motivación de logro puede permitir realizar predicciones o anticipar las reacciones ante un éxito o un fracaso, y proporciona una ventana hacia el conocimiento de las respuestas cognitivas y conductuales ante la evaluación (Weiner, 2000).

Por su parte, parece claro que la motivación de logro está relacionada con el tipo de tarea que se desempeña y con la dificultad de la misma (Capa, Audiffren y Ragot, 2008). Particularmente, la cantidad de esfuerzo empleado en la realización de una tarea es predicho por la percepción de la dificultad de la tarea, y cuanto mayor sea la percepción subjetiva de la dificultad de la tarea mayor será el esfuerzo empleado para la realización de la misma (Capa et al., 2008). Relacionado con esto, Locke y Latham (2002) establecieron que las metas consideradas difíciles conducen a mayores índices de esfuerzo en la consecución de las mismas que las que son consideradas como fáciles.

Desde un punto de vista más global en lo referente a la teoría de la motivación, se debe tener en cuenta que las personas difieren entre sí, y responden de distinta forma ante las metas propuestas; es a partir de ciertos estándares individuales asumidos, por los que se determinan lo que supone un reto, y lo que es o no recompensa, además de la interpretación que se dé a esa recompensa. Debido a lo anterior, Gómez-Miñambres (2012) describe la existencia de dos vías en los procesos de motivación en el trabajo de las personas. Una primera vía en donde la motivación proviene a partir de incentivos extrínsecos de diversa índole, monetario, aprobación de los otros etc.; e intrínsecos en donde es el propio individuo quien desarrolla un sentimiento interno de logro en la consecución de una meta.

Por todo lo expuesto hasta aquí, el objetivo de esta investigación consiste en analizar la relación existente entre los niveles motivacionales y la autoeficacia, en una muestra de estudiantes de instrumentos de cuerda frotada de nivel superior en España.

\section{Método}

\section{Participantes}

La muestra está compuesta por 140 estudiantes (58 hombres y 82 mujeres) del Título Superior de Música pertenecientes a 5 centros de formación superior españoles. 
Todos los estudiantes son intérpretes de instrumentos de cuerda frotada (74 violín, 23 viola, 31 violonchelo y 12 contrabajo). El rango de edad de estos estudiantes abarca desde los 17 hasta los 51 años con una edad media de 20.83 $($ D.T. $=3.79)$.

\section{Procedimiento}

Todos los datos fueron recogidos a lo largo de un mismo curso académico in situ por distintos miembros del equipo investigador quienes se desplazaron a los diferentes centros para una mejor adquisición de cuestionarios.

\section{Instrumentos y análisis de datos}

Para el estudio de la motivación se empleó la escala de motivación de logro en contextos musicales (Zarza, 2014). Es un cuestionario de 26 preguntas evaluadas en una escala Likert de 9 puntos en la que el cómputo general de la misma arroja un estadístico de fiabilidad $\alpha$ de Cronbach de 0.775. Presenta en su versión confirmatoria de 18 ítems ( $\alpha$ de Cronbach de 0.774) 5 subescalas diferenciadas: interés (4 ítems), esfuerzo ( 2 ítems), perfeccionismo (2 ítems), tarea (8 ítems) y examen (2 ítems).

Respecto a la escala de autoeficacia, se ha empleado la adaptación realizada por Sanjuán, Pérez y Bermúdez. en el año 2000. El instrumento está constituido por 10 preguntas evaluadas en una escala de 10 puntos y presentó un $\alpha$ de Cronbach de 0.87 .

Relativo al análisis de datos, se han llevado a cabo análisis descriptivos y correlacionales con los programas informáticos SPSS 19.0.

\section{Resultados}

En lo concerniente al aspecto motivacional vemos que la muestra en todos los factores se producen diferencias de medias significativas con respecto a la media teórica del instrumento. En este sentido, para el factor interés $\mathrm{t}=38.725 ; \mathrm{p}=.000$; para el de esfuerzo $\mathrm{t}=19.859 ; \mathrm{p}=.000$; para el de perfeccionismo $\mathrm{t}=-6.821 ; \mathrm{p}=.000 ;$ para el de tarea $\mathrm{t}=3.288 ; \mathrm{p}=.001 \mathrm{y}$ para el de exámenes $\mathrm{t}=8.387 ; \mathrm{p}=.000$. Como se aprecia de mejor modo en la Tabla 1, todos los factores excepto el de perfeccionismo presentan valores superiores a la media teórica del instrumento. 
Tabla 1. Estadísticos descriptivos.

\begin{tabular}{|c|c|c|c|c|c|c|}
\hline & $\mathrm{N}$ & Mínimo & Máximo & Media & $\begin{array}{c}\text { Media } \\
\text { Teórica }\end{array}$ & Desv. típ. \\
\hline Interés & 140 & 20.00 & 36.00 & 31.8802 & 20 & 3.62990 \\
Esfuerzo & 140 & 4.00 & 18.00 & 14.6071 & 10 & 2.74503 \\
Perfeccionismo & 140 & 2.00 & 18.00 & 7.2929 & 10 & 4.70190 \\
Tarea & 140 & 24.00 & 67.00 & 42.7155 & 40 & 9.77112 \\
Exámenes & 140 & 5.00 & 18.00 & 11.9458 & 10 & 2.74518 \\
N válido (según lista) & 140 & & & & & \\
\hline
\end{tabular}

Si analizamos más pormenorizadamente la distribución muestral vemos cómo en el caso del factor interés un $20 \%$ de la población presenta medias inferiores alejadas a la media en más de una desviación típica del factor de interés, un $14.3 \%$ presenta puntuaciones alejadas más de una desviación típica de la media por el extremo inferior en el factor esfuerzo, de manera análoga ocurre con un $20 \%$ de la población para el factor perfeccionismo, en el caso del factor tarea este porcentaje se sitúa en el 14.3\% mientras que para el factor exámenes este porcentaje es del 19.3\%.

En cuanto a las relaciones entre los factores vemos (Tabla 2) que el factor interés presenta correlación positiva y significativa $(\mathrm{r}=.539 ; \mathrm{p}=.000)$ con el factor esfuerzo y negativa con el factor perfeccionismo $(r=-.280 ; p=$ $.001)$. Por su parte, el factor esfuerzo presenta correlación significativa e inversa con el factor perfeccionismo $(\mathrm{r}=-.246 ; \mathrm{p}=.003)$ y directamente proporcional tanto con el factor tarea $(r=.174 ; p=.040)$ como con el factor exámenes $(\mathrm{r}=.196 ; \mathrm{p}=.020)$. A su vez, el factor perfeccionismo presenta correlación significativa con el factor tarea $(\mathrm{r}=.268 ; \mathrm{p}=.001)$ y el factor tarea con el factor exámenes $(\mathrm{r}=.512 ; \mathrm{p}=.000)$.

Tabla 2. Correlaciones.

\begin{tabular}{|c|c|c|c|c|c|}
\hline & Interés & Esfuerzo & Perfeccionismo & Tarea & Exámenes \\
\hline Interés & 1 & $.539^{* *}$ & $-.280^{* *}$ & & \\
\hline Esfuerzo & $.539^{* *}$ & 1 & $-.246^{* *}$ & $.174^{*}$ & $.196^{*}$ \\
\hline Perfeccionismo & $-.280^{* *}$ & $-.246^{* *}$ & 1 & $.268^{* *}$ & .080 \\
\hline Tarea & & $.174^{*}$ & $.268^{* *}$ & 1 & $.512^{* *}$ \\
\hline
\end{tabular}

En lo referente al análisis de la autoeficacia (Tabla 3), vemos cómo el valor medio del sumatorio total de la escala arroja un valor medio (68.71; D.T. $=12.45$ ) superior a la media teórica del instrumento (50 puntos) siendo esta diferencia además estadísticamente significativa $(\mathrm{t}=17.708 ; \mathrm{p}=.000)$.

Tabla 3. Estadísticos descriptivos.

\begin{tabular}{|c|c|c|c|c|c|c|}
\hline & $\mathrm{N}$ & Mínimo & Máximo & Media & Media teórica & Desv. típ. \\
\hline Autoeficacia & 139 & 28.00 & 97.00 & 68.7122 & 50 & 12.45819 \\
N válido (según lista) & 139 & & & & & \\
\hline
\end{tabular}


Sin embargo, podemos ver también en la distribución muestra cómo un $14.4 \%$ de la población presenta puntuaciones medias inferiores a más de una desviación típica de la media muestral (Figura 1).

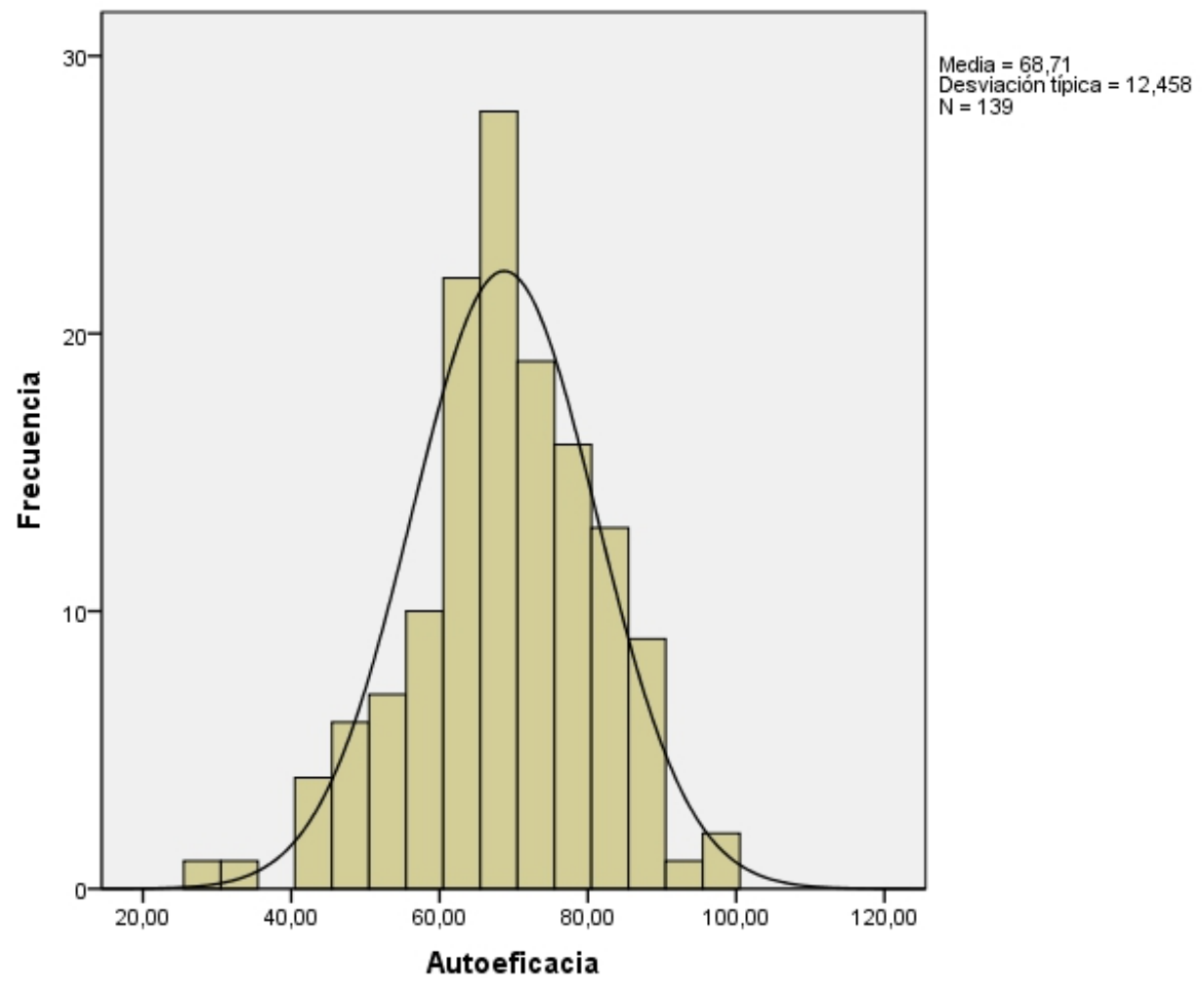

Figura 1. Distribución de la muestra.

Asimismo, comentar que la relación entre los factores motivacionales descritos anteriormente con la autoeficacia de manera correlacional arroja relaciones significativas y directamente proporcionales con los factores esfuerzo $(\mathrm{r}=.167 ; \mathrm{p}=.049)$, tarea $(\mathrm{r}=.566 ; \mathrm{p}=.000)$ y exámenes $(\mathrm{r}=.328$; $\mathrm{p}=.000)$, no siendo significativas $(\mathrm{p}>.05)$ con los factores de interés $\mathrm{y}$ perfeccionismo.

Llegados a este punto, se plantea una regresión lineal por el método enter en la que se intente explicar el factor de autoeficacia a partir de las relaciones descritas anteriormente con el constructo motivacional y sus distintos factores. Así, llegamos a un modelo significativo $(\mathrm{F}=12.930 ; \mathrm{p}=$ $.000 ; \mathrm{D}-\mathrm{W}=1.838 ; \mathrm{R}^{2}=.302$ ) en el que se consigue explicar el $30.2 \%$ de la varianza de la autoeficacia y en el que sólo toma presencia significativa en el modelo el factor motivacional de la tarea $(\mathrm{t}=6.209 ; \mathrm{p}=.000 ; \beta=.542)$ mientras que no aportan carga explicativa significativa los factores de interés $(\mathrm{t}=-.011 ; \mathrm{p}=.991)$, esfuerzo $(\mathrm{t}=.616 ; \mathrm{p}=.539)$, perfeccionismo $(\mathrm{t}=-.405$; $\mathrm{p}=.686)$ y exámenes $(\mathrm{t}=.530 ; \mathrm{p}=.597)($ Tabla 4$)$. 
Tabla 4. Regresión. Variable dependiente Autoeficacia.

\begin{tabular}{|c|c|c|c|c|c|c|c|}
\hline \multicolumn{8}{|c|}{ Variable dependiente Autoeficacia } \\
\hline & $\begin{array}{c}\text { Coeficientes } \\
\text { tipificados }\end{array}$ & \multirow[t]{2}{*}{$\mathrm{t}$} & \multirow[t]{2}{*}{ Sig. } & \multicolumn{2}{|c|}{$\begin{array}{l}\text { Estadísticos de } \\
\text { colinealidad }\end{array}$} & & \\
\hline & Beta & & & Tolerancia & FIV & $\mathrm{F}$ & Sig \\
\hline Interés & -.001 & -.011 & .991 & .680 & 1.470 & \multirow{5}{*}{12.930} & \multirow{5}{*}{.000} \\
\hline Esfuerzo & .054 & .616 & .539 & .670 & 1.491 & & \\
\hline Perfeccionismo & -.032 & -.405 & .686 & .809 & 1.236 & & \\
\hline Tarea & .542 & 6.209 & .000 & .663 & 1.508 & & \\
\hline Exámenes & .044 & .530 & .597 & .729 & 1.372 & & \\
\hline
\end{tabular}

\section{Conclusión}

Se ha podido evidenciar cómo relacionan los constructos de motivación y autoeficacia. Sin embargo, ahondado más en esta relación y disgregando los distintos factores motivacionales (interés, esfuerzo, perfeccionismo, tarea y exámenes) descritos por Manassero y Vázquez (1998) y con la práctica y el estudio musical (Zarza, 2014) vemos cómo no todos ellos se relacionan del mismo modo con la autoeficacia. Así el papel de los factores de interés y perfeccionismo y su relación con la autoeficacia no ha aportado datos concluyentes, mientras que sí que lo hacen y de manera directamente proporcional los factores de esfuerzo, tarea y exámenes. Sin embargo, estas relaciones han quedado subsumidas en la fuerte relación que presenta la autoeficacia con las demandas específicas del componente motivacional de la tarea de cara a forjar o sostener el sentimiento de autoeficacia. Estos resultados son acordes con investigaciones anteriores (Orejudo, Zarza, Casanova, Rodríguez y Mazas, 2017) en donde se ve también cómo las demandas específicas de la tarea resultan clave en la mediación con la autoeficacia, así como en la relación con otros constructos tan importantes en la práctica de la música como pueda ser la ansiedad escénica.

En este sentido se hace necesaria más investigación que siga estudiando la relación motivacional con constructos relacionados como puedan ser el optimismo, la sensibilidad al premio y al castigo o incluso determinadas estrategias pedagógicas y estilos de afrontamiento del estudio musical. Toda esta reflexión contribuirá a la mejorará del proceso de enseñanza-aprendizaje musical.

\section{References:}

1. Atkinson, J. W. (1964). An Introduction to Motivation. Princeton, N. J.: Van Nostrand.

2. Bandura, A. (1986). Social foundations of thought and action: A social cognitive theory. Englewood Cliffs, NJ: Prentice-Hall.

3. Capa, R. L., Audiffren, M., \& Ragot, S. (2008). The effects of achievement motivation, task difficulty, and goal difficulty on 
physiological, behavioral and subjective effort. Psychophysiology, 45(5), 859-868. doi:10.1111/j.1469-8986.2008.00675.x

4. Elias, H., Mustafa, S. M. S., Roslan, S., \& Noah, S. M. (2011). Motivational predictors of academic performance in end year examination. Procedia - Social and Behavioral Sciences, 29, 11791188.

5. Gómez-Miñambres, J. (2012). Motivation through goal setting. Journal of Economic Psychology, 33, 1223-1239.

6. Grenn, J., Liem, G. A. D., Martin, A. J., Colmar, S., Marsh, H. W., \& McInerney, D. (2012). Academic motivation, self-concept, engagement, and performance in high school: Key processes from a longitudinal perspective. Journal of adolescence, 35, 1111-1122.

7. Heider, F. (1958). The psychology of interpersonal relations. New York, NY: Wiley.

8. Hsieh, P. H. P. \& Schallert, D. L. (2008). Implications from selfefficacy and attribution theories for an understanding of undergraduates' motivation in a foreign language course. Contemporary Educational Psychology, 33, 513-532.

9. Locke, E. A. \& Latham, G. P. (2002). Building a practically useful theory of goal setting and task motivation: A 35-year odyssey. American Psychologist, 57(9), 705-717. doi:10.1037/0003066X.57.9.705

10. Manassero, M. A. \& Vázquez, A. (1998). Validación de una escala de motivación de logro. Psicothema, 10(2), 333-351.

11. Marsh, H. W. (2007). Self-concept theory, measurement and research into practice: The role of self-concept in educational psychology. Leicester, UK: British Psychological Society.

12. Orejudo, S., Zarza, F. J., Casanova, O., Rodríguez, C., \& Mazas, B. (2017). The relation of music performance anxiety (MPA) to optimism, self-efficacy, and sensitivity to reward and punishment: Testing Barlow's theory of personal vulnerability on a sample of Spanish music students. Psychology of Music, 45(4), 570-583. doi:10.1177/0305735616674791

13. Pintrich, P. R. (2003). Motivation and classroom learning. In W. M. Reynolds, G. Miller, \& I. B. Weiner (Eds.) (2003), Handbook of psychology: Educational psychology, Vol. 7 (pp. 103-122). Hoboken, NJ: John Wiley \& Sons.

14. Sanjuán, P., Pérez, A. M., \& Bermúdez, J. (2000). Escala de autoeficacia general: datos psicométricos de la adaptación para la población española. Psicothema, 12(2), 609-613.

15. Skinner, E. A., Kindermann, T. A., Connell, J. P., \& Wellborn, J. G. (2009). Engagement as an organizational construct in the dynamics of 
motivational development. In K. Wentzel \& A. Wigfield (Eds.), Handbook of motivation in school (pp. 223-245). Malwah, NJ: Erlbaum.

16. Spinath, B., Spinath, F. M., Harlaar, N., \& Plomin, R. (2006). Predicting school achievement from general cognitive ability, selfperceived ability, and intrinsic value. Intelligence, 34, 363-374.

17. Weiner, B. (1976). Attribution theory, achievement motivation, and the educational process. Review of Educational Research, 42, 203-215.

18. Weiner, B. (2000). Interpersonal and intrapersonal theories of motivation from an attributional perspective. Educational Psychology Review, 12, 1-14.

19. Zarza, F. J. (2014). Variables psicológicas y pedagógicas como predictoras de la ansiedad escénica en estudiantes de Grado Superior de Música de España. Tesis doctoral. Zaragoza, Spain: Universidad de Zaragoza. 